INPLASY

PROTOCOL

To cite: Ma et al. Acupotomy Combined with Massage for cervical spondylosis of vertebral artery type : a protocol for systematic review and meta-analysis. Inplasy protocol 202210035. doi: 10.37766/inplasy2022.1.0035

Received: 08 January 2022

Published: 08 January 2022

Corresponding author: Dongyang $\mathrm{Ma}$

414694594@qq.com

Author Affiliation:

Changchun University of

Chinese Medicine.

Support: International Fund for nature.

Review Stage at time of this submission: Preliminary searches.

Conflicts of interest:

None declared.

\section{Acupotomy Combined with Massage for cervical spondylosis of vertebral artery type : a protocol for systematic review and meta-analysis}

Ma, DY1; Guo, C2; Li, ZX33:Qian, X4; Li, XF5; Liu, JY6; Gnag, ZH7; Qi, W8.

Review question / Objective: Cervical spondylosis of vertebral artery type (CSA) is a clinical syndrome of dizziness and nausea. Both Massage and Acupotomy have been widely used in the treatment of CSA, in China and achieved satisfied efficacy. Therefore, the aim of this study is to systematically evaluate the clinical efficacy of acupotomy combined with massage in the treatment of CSA.

Condition being studied: Acupotomy Combined with Massage for cervical spondylosis of vertebral artery type. We used the Review Manager 5.4 software provided by the Cochrane Collaborative Network for statistical analysis.

INPLASY registration number: This protocol was registered with the International Platform of Registered Systematic Review and Meta-Analysis Protocols (INPLASY) on 08 January 2022 and was last updated on 08 January 2022 (registration number INPLASY202210035).

\section{INTRODUCTION}

Review question / Objective: Cervical spondylosis of vertebral artery type (CSA) is a clinical syndrome of dizziness and nausea. Both Massage and Acupotomy have been widely used in the treatment of CSA, in China and achieved satisfied efficacy. Therefore, the aim of this study is to systematically evaluate the clinical efficacy of acupotomy combined with massage in the treatment of CSA.

Condition being studied: Acupotomy Combined with Massage for cervical spondylosis of vertebral artery type. We 
used the Review Manager 5.4 software provided by the Cochrane Collaborative Network for statistical analysis.

\section{METHODS}

Participant or population: (1) Patients are more common in the middle-aged and elderly, with chronic onset, mild and severe, and recurrent attacks. (2) The patient had a history of sudden collapse with cervical vertigo.

Intervention: Acupotomy combined with Massage.

\section{Comparator: Non drug treatment.}

\section{Study designs to be included: RCT.}

Eligibility criteria: (1) Patients are more common in the middle-aged and elderly, with chronic onset, mild and severe, and recurrent attacks. (2) The patient had a history of sudden collapse with cervical vertigo. (3) The neck rotation test was positive, and the head and neck movement was occasionally accompanied by symptoms such as dizziness and nausea. (4) The X-ray film of the neck showed degenerative change of the neck and hyperplasia of the uncinate vertebral joint. (5) Patients are often accompanied by sympathetic symptoms. (6) Ocular and otogenic vertigo were excluded.

Information sources: We searched the China National Knowledge Infrastructure, Chinese Scientific Journal Database, Wanfang Database, China Doctoral Dissertations Full-Text Database, China Master's Theses Full-Text Database, Cochrane Central Register of Controlled Trials, PubMed, and Embase.

Main outcome(s): Recovery: the original headache, dizziness and other clinical symptoms completely disappear, and can study and work normally; Improvement: the original clinical symptoms such as headache and vertigo basically disappeared, and the patient occasionally had acid swelling of neck and shoulder;
Ineffective: no improvement in clinical symptoms.

Quality assessment / Risk of bias analysis: Cochrane.

Strategy of data synthesis: We will use Review Manager 5.4, provided by the Cochrane Collaborative Network for statistical analysis.

Subgroup analysis: People over 18 years old and less than $\mathbf{7 0}$ years old.

Sensitivity analysis: Stata software was used for sensitivity analysis to reflect the sensitivity of articles by the change of effect quantity after deleting one of them.

Country(ies) involved: China.

Keywords: Acupotomy; Massage; cervical spondylosis of vertebral artery type; protocol.

Contributions of each author:

Author 1 - Dongyang Ma.

Author 2 - Chao Guo.

Author 3 - ZhongXU Li.

Author 4 - Xin Qian.

Author 5 - XueFeng Li.

Author 6 - JiaYI Liu.

Author 7 - ZhaoHUI Wang.

Author 8 - Wei Qi. 